\title{
Motivation and Ways of Improving biomedical Translation Ability of Foreign Nursing Students in Medical Colleges under the Epidemic Situation
}

\author{
Haiyan Zhan ${ }^{a,}$, Wenjing Han ${ }^{b}$ and Jiaxin $\mathrm{Hu}^{\mathrm{c}}$ \\ Nusing School, Tianjin University of Traditional Chinese Medicine, Tianjin 301617, China \\ ahy14795228727@outlook.com, b944688214@qq.com, c455316698@qq.com \\ *Corresponding author
}

Keywords: Foreign nursing major; biomedical translation; Motivation; English teaching.

\begin{abstract}
With the acceleration of globalization, the cross-border exchange of knowledge and talents is becoming more frequent, and nurses have more opportunities to enter the international market for employment. Nursing English, a branch of English for Special Purpose, is a professional English for nurses. Aiming at the cultivation of students' learning motivation in the teaching process, this paper systematically studies the motivation and ways of improving biomedical translation ability of foreign nursing students in medical colleges under the epidemic situation. From the aspects of word translation, text translation, medical culture transmission, etc., this paper expounds the specific measures of training medical students' professional English translation skills and analyzes the influence of various learning motives on improving biomedical translation ability, in order to provide scientific and reasonable suggestions for foreign nursing professional English teaching in China.
\end{abstract}

\section{Introduction}

With the rapid development of medical science and technology, medical knowledge is changing with each passing day, and new technologies and new therapies are constantly emerging [1]. Biomedicine and humanities permeate and merge with each other, forming a new frontier discipline, which has brought unprecedented changes and development. In the process of English teaching, people pay more and more attention to the factors of learners, and the research on language learning pays more and more attention to the differences of learners' personal characteristics. Cross-border exchange of knowledge and talents has become increasingly frequent, and the demand for high-level nursing talents in the international market has increased [2]. Psychology divides motivation into internal motivation and external motivation. Internal motivation is the internal force of learners, which can stimulate their subjective wishes and strive to achieve learning goals; External motivation is the motivation induced by external conditional stimulation [3]. As an important factor, motivation is the premise of other factors, and it is one of the decisive factors to learn English well. Therefore, this paper discusses the motivation and ways of improving biomedical translation ability of foreign nursing students in medical colleges under the epidemic situation in China.

\section{Motivation and its Function}

Motivation is the internal psychological process or internal motivation to stimulate and maintain individual activities under the guidance of goals. Motivation is an internal psychological process, which can be inferred from tasks selection, effort, persistence of activities and verbal expression [4]. Motivation is not used to explain what the activity itself is, but to explain why a person is engaged in such an activity, to explain why a person thinks or does so. biomedical translation teaching is an important way to cultivate students' language comprehension and expression ability. Translators have formed certain aesthetic cultivation, values, artistic preferences and creative talents in their own social life. Therefore, translators' translation activities are often selective rather than indiscriminate 
[5]. Therefore, the applicability of appropriateness pragmatic principle in translation is self-evident. Especially foreign-related industries need practical talents who can communicate effectively in professional English at work. The following two points should be paid attention to in cultivating students' learning purpose: 1 . Students should be guided to combine personal purpose with social purpose. 2. Long-term learning objectives should be combined with short-term learning objectives.

Students' learning motivation comes from needs, and needs are the source of students' learning enthusiasm. The essence of the formation and development of students' learning motivation is the arousal and formation of psychological needs, and it is a process in which psychological needs and goals that can satisfy them are interrelated. The main purpose of contrastive analysis is to try to compare the characteristics of two languages, emphasize the differences between different languages, reveal and predict the possible difficulties in foreign language learning, and reduce the interference and differences between the two languages. Whether a text can be selected depends on whether the translator is interested in the text, that is, whether the translator can correctly interpret the content, characteristics, charm and style of the selected text and resonate with it depends on the translator's own needs. Therefore, "faithfulness" is the foundation and premise of biomedical translation, while "fluency" is a means and way to achieve perfection in biomedical translation [6].

\section{Needs Analysis of Foreign Nursing Learners}

\subsection{Analysis of foreign nursing students}

There are mainly two kinds of students: high school graduates who failed to be admitted to ordinary colleges and universities in the college entrance examination, and junior high school graduates who took the 3+2 examination. Generally speaking, students are between 15 and 21 years old and have studied English for 6 to 9 years, but their English foundation is relatively weak. biomedical translation requires translators to have a certain reserve of professional knowledge. biomedical field involves the knowledge of medicine, biology, statistics, etc. The contents of medical literature include all kinds of experimental design, chart display and experimental results. The vast majority of foreign-related nursing vocational students are afraid or resistant when they first come into contact with medical English, and feel that these articles are like "heavenly books", which is difficult to start. In view of these characteristics, appropriateness in EST translation is faithful and fluent. Faithfulness means to express the ideological content of the original work completely and accurately, while maintaining the original style. At this level, English teaching does not need to consider students' learning motivation, major and future employment, and teachers only need to teach students general English language knowledge [7]. However, there is no obvious gender difference in English learning of foreign nursing students, which is due to the particularity of nursing major. There is a big difference between male and female students, and there are only a few boys or even none in many classes. We should focus on medical terms, medical article genres, medical literature reading, how to translate medical literature and so on, and pay attention to cultivating students' language skills.

\subsection{Analysis of learning purpose}

With the continuous progress in the medical field, new drugs and new therapies emerge one after another, and international exchanges in this field are increasing day by day, thus stimulating the market demand for biomedical translation. Teachers should point out to students that medical English vocabulary is different from ordinary English vocabulary in word formation. Relatively speaking, the former is more regular and needs to pay more attention to the mastery of word formation components. The translator's task is to convey this objective existence intact to non-original readers. Although translation is a re-creation of language, it is essentially different from pure creation. On the basis of the existing English, through the study of nursing English, students can establish a professional English vocabulary system, acquire communicative language in professional fields, practice professional article writing paradigm, and meet the needs of future work. However, at present, the teaching staff of foreign nursing English is relatively weak, and the construction of 
teaching staff lags behind. Many colleges and universities take full-time English teachers as their first choice, but English teachers don't know much about nursing. It is known that some medical colleges and universities start professional medical English translation training one year before graduation, while some colleges and universities have not yet opened, which is far from the actual needs of students in future work [8]. The theoretical knowledge of English translation is not enough to combine basic English and nursing English organically to translate high-quality professional articles.

English learning motivation includes internal motivation and external motivation. The root causes of the two motives are also different. Intrinsic motivation comes from an individual's interest in what he is doing, while external motivation is the result of external factors. The internal and external motivation of English learning can stimulate learners to take English learning activities. Therefore, nurses not only need to have a certain foundation of public English, but also need to master the communicative skills of nursing English, that is, listening, speaking, reading, writing and translating skills. In the explanation of texts, students are often organized to make syntactic analysis of long sentences and difficult sentences, so that students can grasp the English sentence structure from a macro perspective. A qualified medical translator should not only have skillful translation skills, but also have a certain degree of understanding of the medical knowledge involved in the translated text. Otherwise, the biomedical translation project cannot be started. Strict language and professional requirements of biomedical translation have greatly raised the threshold of entry in this field. Teachers can analyze word-formation components with students and provide examples to consolidate them when teaching medical terms. After repeated contact with the corresponding vocabulary, students naturally master these word-forming elements. Generally speaking, each style has different corpora and expression means to express its own characteristics, that is, it often has its own special words, special syntactic structure and text structure, language style and so on, which is an important point that distinguishes this style from other styles.

\section{Ways to Cultivate biomedical Translation Ability of Foreign Nursing Majors}

\subsection{Vocabulary translation training}

The structure of English language is hierarchical (Figure 1)[9]. Medical literature often uses preposition short comments, participle phrases, master-slave complex sentences and parallel complex sentences, which are lengthy and complex in sentence structure. Therefore, besides training students to master the skills of word translation, they should also improve their skills of handling sentences.

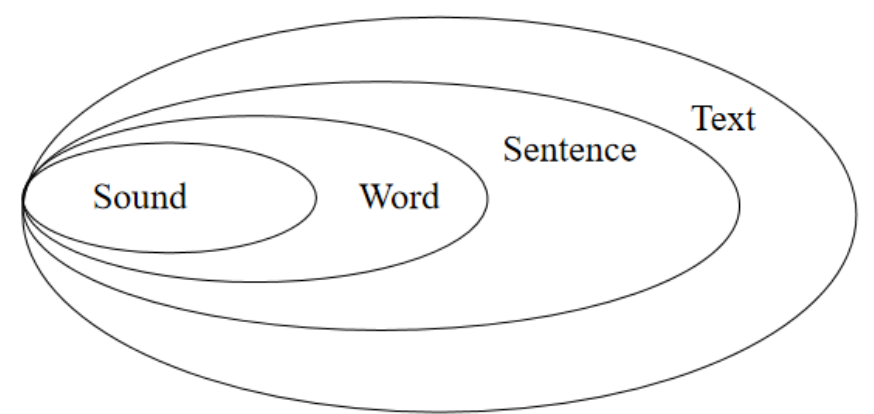

Figure 1. The hierarchical structure of English language

The word-formation methods of medical professional terms are similar to those of general English, and they are often produced by means of suffix, derivation and compound. The affixation method, synthesis method and abbreviation method are common, and most of the vocabulary is polysyllabic and morpheme. Translation requires translators to have strong abilities in reading, writing and translation, while interpretation requires translators to have comprehensive abilities in listening, speaking, reading, writing and translating. Expand students' knowledge, improve their enthusiasm for learning English, and stimulate students' enthusiasm and enthusiasm for going out. In the teaching process of nursing English, the direct communicative activities can be listening to the 
conversation between doctors and patients, asking about the patient's illness, reading the drug instructions or the original nursing textbook. By comparison, students can not only clearly recognize the differences in sentence structure between English and Chinese, such as word order differences, English parataxis, Chinese parataxis, etc., and improve their sentence structure analysis ability. In order to accurately convey the core content of the source language text, it is necessary to find the commonly used and professional expressions recognized by the biomedical community. It is not allowed to use new words arbitrarily. When encountering medical professional vocabulary, we can "read the words according to the text, read them at will", and can "draw inferences from one instance and bypass by analogy", which will be of great benefit to students' scientific memorizing of medical vocabulary and reading of professional articles.

\subsection{Listening and speaking teaching}

Listening and speaking are the most intuitive embodiment of communicative competence. The syllabus of English for foreign nursing clearly proposes to cultivate students' ability to communicate in English. In class, teachers and students should respect each other first, and then add interesting learning links, which can promote students' learning by playing English songs or movies; Because there are differences between Chinese and English thinking modes, sometimes even quite different, we should pay attention to the differences in thinking when translating, and convey the information of the original with the expression of the target language. English teaching for foreign nursing majors should not only be based on language learning, but also pay attention to professional knowledge learning. In nursing English teaching, teachers should choose topics closely related to nurses' work, and the closer they are to the real situation, the more practical they are. When explaining the text, we should focus on helping students understand the knowledge that plays an important role in discourse understanding, such as substitution, ellipsis, cohesion, etc., and train students to judge the logical relationship between sentences and the cohesive devices of discourse coherence. Noun translation often involves the accurate translation of terms. Other words, such as verbs, generally avoid colloquial words. In translating these verbs, we should abandon general words and weigh professional expressions. The combination of translation and interpretation can strengthen the interaction between teachers and students, cultivate students' awareness of autonomous learning and cooperative learning, and attach importance to classroom translation practice, such as many times of simulated conference on-site interpretation exercises, translation of excellent abstracts and so on.

\subsection{Communicative translation is dominant}

From the sentence level, biomedical literature mostly contains nominalization structure and impersonal subject, and appears in the form of long complex sentences, parallel structure, ellipsis, inversion and non-predicate structure. Therefore, before class, teachers should learn about students and communicate with relevant professional teachers to know what professional knowledge students have learned and what professional knowledge they have not yet touched. Therefore, it is necessary to carefully select several synonymous and structurally different language forms in the target language in terms of words, phrases, sentences, paragraphs, etc., and the selection is not only related to language structure factors (such as parts of speech, word collocation, context), but also related to style. The purpose of reading teaching for foreign nursing majors is to train students to read English materials related to nursing majors and obtain the required information. The passive voice makes the writing appear objective, which makes the reader pay attention to the described things, phenomena or processes. The basic part of translation should systematically explain the development, background, principles, specific methods and skills of translation, and properly supplement the knowledge of transnational culture and the general situation of nursing in British and American countries, focusing on cultivating students' shorthand and English-Chinese translation ability. Outside the classroom, activities such as English recitation contest, English song contest and English corner can stimulate students' learning enthusiasm and create a harmonious teaching atmosphere. 


\subsection{Stimulation of motivation in teaching process}

Enhance students' interest and stimulate students' motivation to learn English for foreign nursing majors. English teaching in foreign nursing education must be considered from the unique characteristics of foreign nursing education. Teachers can patrol between groups, help students at any time, and ensure the normal discussion. In order to effectively guide students' translation practice and improve their translation ability, some basic translation theories and skills are explained in combination with the relevant teaching materials. It makes the target sentences more fluent, straightforward and close to the language habits of the target language, thus producing the same expression effect for the target readers as the original readers. There are many advantages in designing some scenes that can not only use the current knowledge, but also connect with clinical practice: it can stimulate students' interest and attract students to participate in activities; The choice of synonymy means of sentences should meet the requirements of theme, adapt to the style of writing, suit the style of writing and comply with the harmony of sound and rhyme [10]. Teachers should guide students to read professional-related documents, books and various applied articles, and make students familiar with the basic structural features of medical articles through a large number of readings. Standardized and scientific management and curriculum are important guarantees of teaching quality. However, after all, learning takes learners as the main body, and besides providing a good environment and scientific curriculum, it is more important to rely on learners themselves.

Most students want to improve their English listening and speaking ability in order to meet their own needs, which is an internal learning motivation. As a public required course, teaching is often a large class in which students of various majors and different levels are mixed together. Corpus can expand our reference range to a certain extent, make up our English sense and help improve the quality of translation. Therefore, teachers can try to provide students with the opportunity to experience and practice English, and the content should be closely related to nursing major. It can take various forms, such as case discussion, group activities and role playing. Passive voice has great room for adjustment in its structure and avoiding mentioning performers is conducive to highlighting important concepts, problems, facts, conclusions, etc., and expanding noun phrases. At the same time, biomedical translation training at the corresponding level is carried out, and the translated content is evaluated and analyzed with the strength and method of mutual evaluation by students and teachers. At the same time, biomedical translation training is carried out at the corresponding level, and the translated content is evaluated and analyzed by the force and method of students' and teachers' joint evaluation.

\section{Summary}

Students should learn to create a favorable environment and improve their biomedical translation ability through their own efforts in the limited resources environment. Through the training of translation skills of words, words and texts in medical English, students are required to master a large number of expressions of medical terms and understand the latest medical information. Learning motivation plays an important role in English teaching. Paying attention to the stimulation and cultivation of students' learning motivation, students' learning enthusiasm and initiative will inevitably be enhanced, and the classroom effect will also be improved accordingly. Communicative approach can stimulate students' learning motivation, improve students' learning enthusiasm, improve students' test scores of nursing English, and enhance students' language communication ability in real language environment. In biomedical translation, we can first analyze the characteristics of English and Chinese languages and consciously apply these characteristics, so that the translation will be more in line with English language habits and expressions. 


\section{References}

[1] Qian Xuejuan. The relationship between English learning motivation and strategies and English performance of foreign nursing majors in vocational schools[J]. Business Situation, Vol. 000 (2019) No. 008, p. 266-268.

[2] Lu Xiaojuan, Yang Shaolong, Dong Zhongsheng, et al. Exploration and Practice of Bilingual Teaching of Medical Immunology and Pathogenic Biology in Foreign Nursing Specialty[J]. China Higher Medical Education, (2015) No. 06, p. 67-68.

[3] Pan Yiting. Research on English Vocabulary Teaching of Foreign Nursing Specialty under Narrative Teaching Method[J]. Overseas English (Part 1), Vol. 000 (2019) No. 002, p. 50-52.

[4] Su Yuhuan. Problems and exploration of bilingual teaching of biochemistry in foreign nursing majors in vocational colleges [J]. Health Vocational Education, Vol. 034 (2016) No. 015, p. 13-14.

[5] Wang Lei, Wang Yingjie. A survey of the current situation of nurses carrying out multicultural nursing in foreign medical institutions [J]. Journal of Nursing, Vol. 22 (2015) No. 20, p. 45-48.

[6] Zeng Liping, Wang Lei, Shu Anli. Exploring the bilingual teaching model of "Pathology" course for foreign-related nursing majors[J]. China Health Nutrition, Vol. 26 (2016) No. 027, p. 34-35.

[7] Tang Xiaomei. Exploring the teaching model of listening and speaking English for foreign nursing majors[J]. Journal of Contemporary Teaching and Research, Vol. 060 (2018) No. 12, p. 15-16.

[8] ability survey of domestic nursing students from 2005 to 2014 [J]. Nursing Research, Vol. 30 (2016) No. 004, p. 486-489.

[9] Wang Lei, Wang Yingjie. A survey of the current situation of nurses carrying out multicultural nursing in foreign medical institutions [J]. Journal of Nursing, Vol. 000 (2015) No. 020, p. 41-44.

[10] Wang Kun, Jing Ran, Han Fangying. A Study on the Translation of Conversational Implicature of Film and Television Works from a Cross-cultural Perspective [J]. Frontier Economy and Culture, Vol. 000 (2019) No. 004, p. 103-104. 